\title{
3D Analytical TEM Approach to Effectively Characterize 3D-FinFET Device Features in Semiconductor Wafer-foundries
}

\author{
Wayne Zhao, Stephen Mongeon, Bianzhu Fu, Esther (PY) Chen, Daniel Flatoff, Nicolas LaManque, and Jeremy Russell
}

Engineering Analysis - Physical, Technology Development \& Yield Engineering, Technology Development, GLOBALFOUNDRIES, Malta, New York, USA.

Introductions of 3-Dimentional FinFET transistors in semiconductor device open a new era for continuous shrinkage of semiconductor transistor nodes. One big challenge for physical failure analysis (PFA) and transmission electron microscopy (TEM) is that, for device of 20nm, $14 \mathrm{~nm}$, an beyond, features of interests (FOI) are mostly thinner than a thickness of a TEM lamella (say, around 50 150nm), depending on the orientation. This adds in ambiguity in interpretation of the TEM imaging straightforward. Combined with intrinsic complexity from the 3D-FinFET structures themselves, PFA/TEM teams at waferfoundries truly have to constantly think out of box for novel approaches to effectively and timely isolate faults and conclusively identify root-causes of yield detractors [1 4].

One novel approach, to accommodate aforementioned complicated PFA / TEM cases on 3D FinFET structures, is to introduce 3D TEM characterization by incorporating several traditional TEM routines, without prolonging turnaround. Basically, there are three steps. Step-I, machine a planar view (top-down) TEM lamella with the FOIs in the central region, by focused ion beam (FIB) lift-out. Most importantly, the TEM lamella has to be kept relatively thick on purpose, to avoid prematurely remove layers with defects. Step-II, employ x-ray energy dispersive spectroscopy (XEDS) elemental mapping in an Analytical TEM to explore and pin-point the abnormality. Step-III, extract another cross-sectional TEM lamella right at the location of the abnormal region in the existing thick planar-view TEM lamella, with desired orientation by FIB lift-out. Thanks to technology development of new XEDS hardware, nowadays, fault-isolations by XEDS mapping can be done in $5 \sim 10$ minutes, e.g., by a Super-X detector, which eliminated the concerns of turnaround time and potential artefacts due to sample-drifting.

Demonstrated here is one successful example for the effectiveness of this novel best practice of 3D TEM. The PFA case was for a testing device from a wafer processed during a CMP tool-qualification, back to $1^{\text {st }}$-Si stage of 14XM 3D FinFET technology ramp-up. Figure-1 is a schematic view of 3D FinFET structure. Figure-2a is a planar view TEM image, and Fig$2 \mathrm{~b}$ displays the corresponding XEDS elemental mapping, with overlay of elements, Ti, Hf, and $\mathrm{W}$. If without the navigation from the top-down view XEDS mapping, the pinching of $1^{\text {st }}$ Fin, and distinctions of Ti-rich / Ti depletion regions could have be easily overlooked or even cut through during the progressive FIB, if only by traditionally cross-sectional TEM all the times. Figure-3 is a FIB image illustrating where the additional cross-sectional TEM lamella was extracted from the first planar-view TEM lamella. Figures 4(a) and 4(b) are TEM, and overlay of HAADF-STEM with XEDS mapping on the crosssectional TEM lamella, which validated the pinch of the $1^{\text {st }}$ FIN, and revealed over-polishing of the FINs / Gates by the CMP process.

References:

[1] W. Zhao, et al., Proc. $38^{\text {th }}$ International Symposium for Testing and Failure Analysis, (2012), pp. 347355.

[2] W. Zhao, et al., Microscopy \& Microanalysis, Vol. 19 (Supplement 2), (2013), pp.902-3.

[3] W. Zhao, Microscopy \& Microanalysis, Vol. 12 (Supplement 2), (2006), pp.1030-31CD.

[4] W. Zhao, Symp. Proc. the Material Research Society, 2002 Fall Meeting, (2002), Vol. 738, pp. G7.15.1-6. 

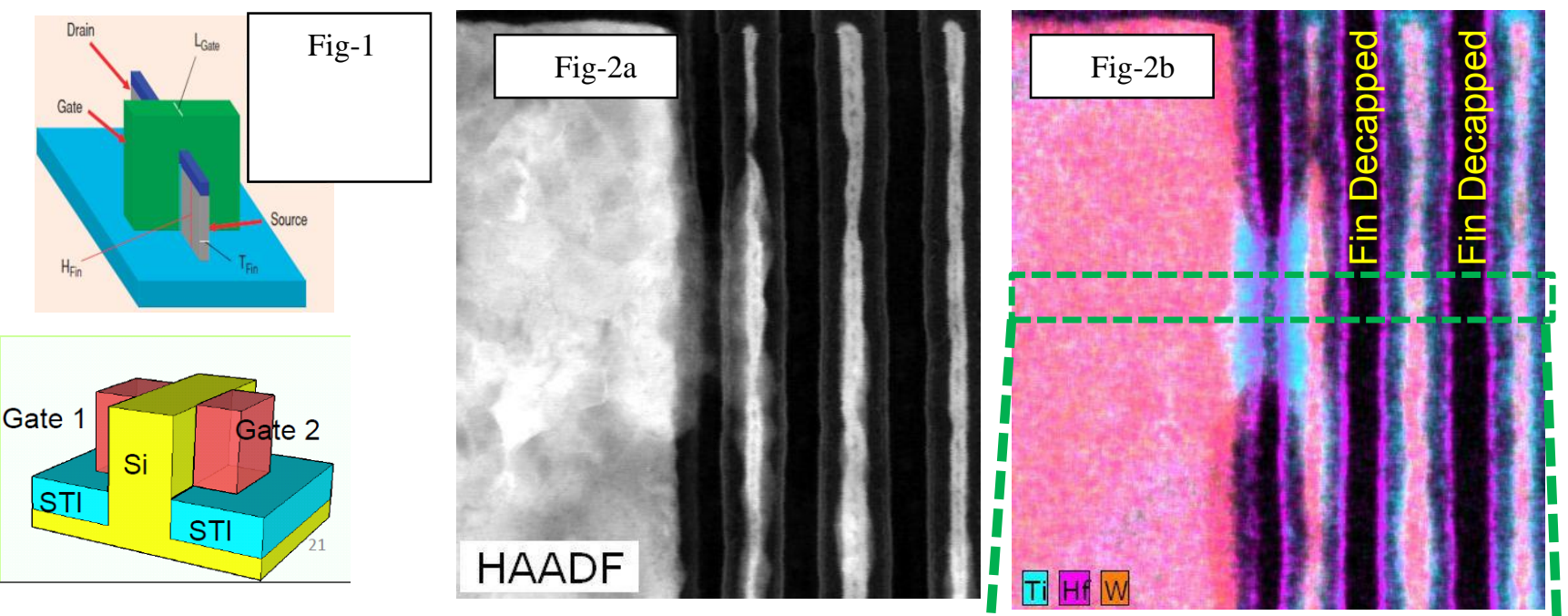

Figure 1. An illustration of 3D FinFET (Courtesy: after Chenming Hu, Aug-2011).

Figure 2. (a) planar-view TEM; and (b) overlay of corresponding XEDS maps of Elemetns, Ti, Hf, W.

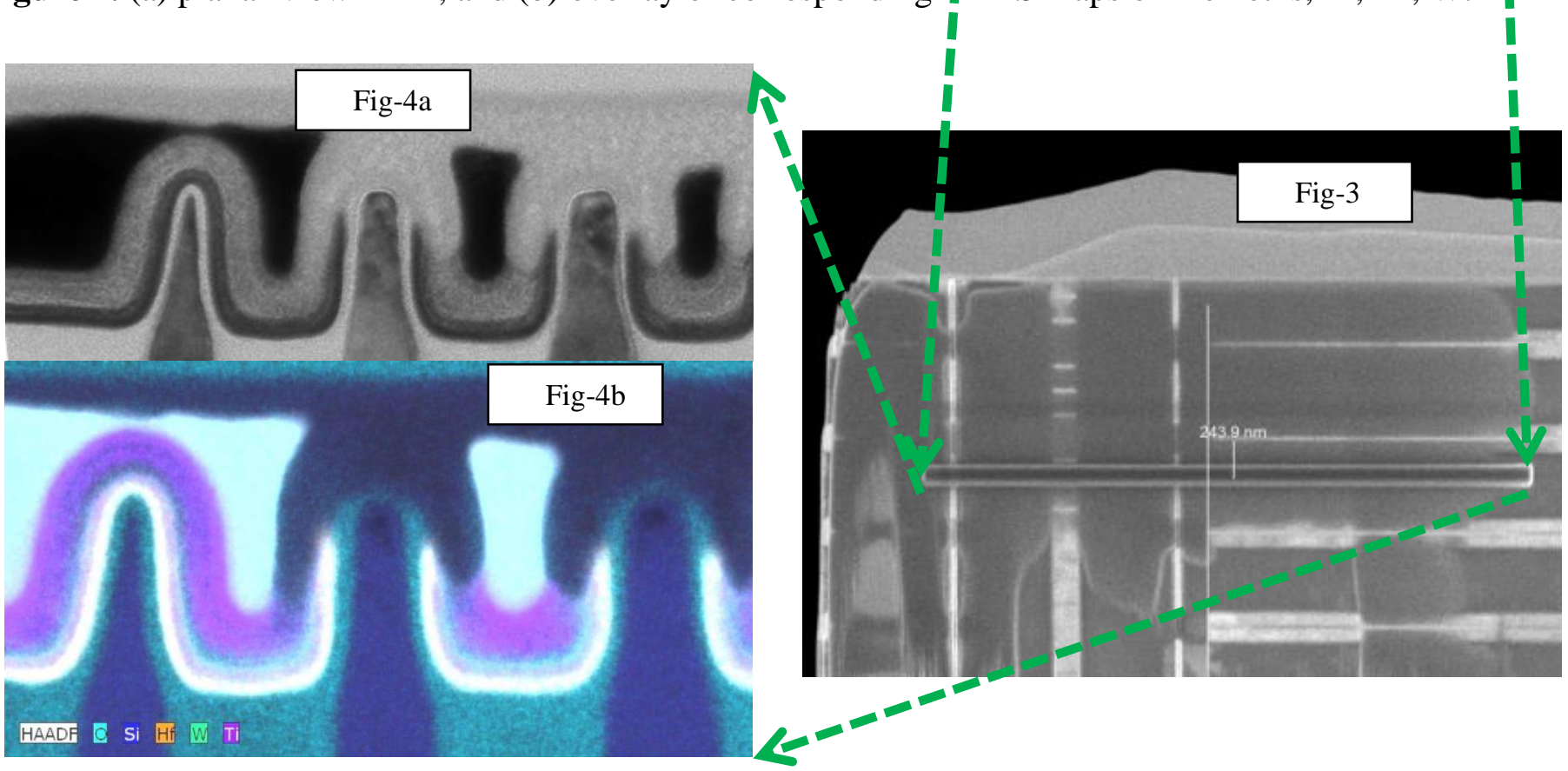

Figure 3. FIB image for the $2^{\text {nd }}$ cross-sectional TEM lamellar on top of the planar TEM lamella being XEDS mapped.

Figure 4. (a) TEM image, and (b) HAADF-STEM image overlay with XEDS elemental maps, from the cross-sectional view TEM lamella. 